\title{
THE RESULTS OF MICROBIAL MUTATION TEST FOR FORTY-THREE INDUSTRIAL CHEMICALS
}

\author{
Hidesuke Shimizu, * Yuji Suzuki,* Nozomi Takemura, * \\ Sumio Goтo** and Hidetsuru Matsushita** \\ 工業化学物質 43 種類の突然変異原性について \\ 清 水英 佑*, 鉿 木勇 司*, 竹村 望* \\ 後藤 純 雄**, 松下秀 鶴**
}

\begin{abstract}
The mutagenicity of 43 industrial chemicals in Salmonella typhimurium (TA98, TA100, TA1535, TA1537, and TA1538) and Escherichia coli (WP2uvrA) was examined. The output of these chemicals in Japan is more than a million kilograms per year. The mutation test was carried out under the condition of absence and presence of rat microsomal activation.

Two chemicals, hexamethylenetetramine and 4,4'-methylenediphenyldiisocyanate, showed mutagenic activity in $S$. typhimurium TA98 and TA100 by metabolic activation. Hexamethylenetetramine also showed mutagenic activity in TA98 without microsomal activation.
\end{abstract}

No mutagenic activity was observed in the 41 chemicals including 4 volatile and gaseous compounds.

\section{INTRODUCTION}

A large number of industrial chemicals have been used as raw materials, organic solvents and intermediates in the production of food additives, drugs, cosmetics, plastics, etc.

Because of its rapidity and economic return, the Salmonella/mammalian microsome test developed in 1975 by Ames et al. ${ }^{1}$ is useful in the chemical industry for screening chemicals for their carcinogenic potential. The mutagenic activity of hundreds of chemicals has been examined by this test, and a close relationship between mutagenicity and carcinogenicity has been demonstrated. ${ }^{2,3)}$ We have tested the mutagenicity of 43 compounds produced as more than a million kilograms per year in Japan in order to estimate the carcinogenic

\footnotetext{
* Department of Public Health, The Jikei University School of Medicine

** The Institute of Public Health Received for publication, July 13, 1985

* 東京慈恵会医科大学公衂衡生学教室

** 国立公愋衛生院 昭和 60 年 7 月 13 日受付
}

risk, and the results are reported herein. Workers in the chemical industry are being exposed to these chemicals, and mutagenicity and/or carcinogenicity of most of them have not been tested so far.

\section{MATERIALS AND METHODS}

Chemicals. The chemical names, structures, sources, diluents, CAS numbers and purity of the 43 chemicals tested are as shown in Tables 1 and 2. The annual output of the 43 chemicals is as shown in Table 3.

Strains. Five strains of Salmonella typhimurium (TA100, TA98, TA1535, TA1537 and TA1538) and a strain of Escherichia coli (WP2uvrA) were used for the mutagenicity test. The strains of $S$. typhimurium were kindly provided by B.N. Ames, U.S.A. and $E$. coli by M. Ishizawa, Japan.

S9 mix. The S9 fraction was prepared from the liver of male Sprague-Dawley rats, weighing about $220 \mathrm{~g}$ (seven weeks old), pretreated with polychlorinated biphenyl (KC 500) at a dose of $500 \mathrm{mg} / \mathrm{kg}$ body weight five days before sacrifice. The $\mathrm{S} 9$ mix contained $0.1 \mathrm{ml}$ of $\mathrm{S} 9 / \mathrm{ml}$.

Mutation test. The mutagenicity of $39 \mathrm{com}-$ 
Table 1. List of 39 compounds tested for mutagenicity.

\begin{tabular}{|c|c|c|c|c|c|c|c|c|}
\hline No. & Name (IUPAC) & Structure & $\underset{(\%)}{\text { Purity }}$ & Diluent & Source & CAS No. $\stackrel{\mathrm{N}}{\mathrm{g}}$ & $\begin{array}{l}\text { Muta- } \\
\text { genicity }\end{array}$ & $\begin{array}{l}\text { Carcino- } \\
\text { genicity } \\
\text { (Ref. No.) }\end{array}$ \\
\hline 1. & Aluminium fluoride & $\mathrm{AlF}_{3}$ & 90 & $\mathrm{H}_{2} \mathrm{O}$ & W & $7784-18-1$ & - & $?$ \\
\hline 2. & $\begin{array}{l}\text { 3-Amino-6-chlorotoluene- } \\
\text { 4-sulfonic acid }\end{array}$ & $\begin{array}{l}\mathrm{C}_{6} \mathrm{H}_{2}\left(\mathrm{CH}_{3}\right)\left(\mathrm{NH}_{2}\right)(\mathrm{Cl})- \\
\quad\left(\mathrm{SO}_{3} \mathrm{H}\right)\end{array}$ & 99.9 & DMSO & $\mathbf{T}$ & $88-53-9$ & - & $?$ \\
\hline 3. & 1-Amino-2-propanol & $\mathrm{CH}_{2} \mathrm{CH}(\mathrm{OH}) \mathrm{CH}_{2} \mathrm{NH}_{2}$ & 97 & $\mathrm{H}_{2} \mathrm{O}$ & $\mathbf{T}$ & $78-96-6$ & - & ? \\
\hline 4. & Ammonium fluoride & $\mathbf{N H}_{4} \mathbf{F}$ & 95 & $\mathrm{H}_{2} \mathrm{O}$ & W & $12125-01-8$ & - & ? \\
\hline 5. & $\begin{array}{l}\text { Ammonium peroxodi- } \\
\text { sulfate }\end{array}$ & $\left(\mathrm{NH}_{4}\right)_{2} \mathrm{~S}_{2} \mathrm{O}_{8}$ & 98 & $\mathrm{H}_{2} \mathrm{O}$ & W & $7727-54-0$ & - & $?$ \\
\hline 6. & Barium chloride & $\mathrm{BaCl}_{8}$ & 99 & $\mathrm{H}_{2} \mathrm{O}$ & $\mathbf{W}$ & $10361-37-2$ & - & $?$ \\
\hline 7. & 2-Butanone & $\mathrm{CH}_{3} \mathrm{COC}_{3} \mathrm{H}_{5}$ & 99 & $\mathrm{H}_{2} \mathrm{O}$ & W & $78-93-3$ & - & $-(7)$ \\
\hline 8. & Butyl acetate & $\mathrm{CH}_{8} \mathrm{COOC}_{4} \mathrm{H}_{9}$ & 99.6 & DMSO & W & $123-86-4$ & - & $?$ \\
\hline 9. & Cerium(IV)dioxide & $\mathrm{CeO}_{2}$ & 99.9 & $\mathrm{H}_{2} \mathrm{O}$ & W & $1306-38-3$ & - & $?$ \\
\hline 10. & Copper(M)oxide & $\mathrm{Cu}_{2} \mathrm{O}$ & 90 & $\mathrm{H}_{2} \mathrm{O}$ & W & $1317-39-1$ & - & $?$ \\
\hline 11. & Decanedioic acid & $\mathrm{HOOC}\left(\mathrm{CH}_{2}\right)_{8} \mathrm{COOH}$ & 99 & DMSO & $\mathbf{T}$ & $111-20-6$ & - & $?$ \\
\hline 12. & 1-Dodecanol & $\mathrm{C}_{12} \mathrm{H}_{25} \mathrm{OH}$ & 90 & DMSO & W & $112-53-8$ & - & $-(35)$ \\
\hline 13. 1 & Dodecylbenzen & $\mathrm{C}_{6} \mathrm{H}_{5}\left(\mathrm{C}_{12} \mathrm{H}_{25}\right)$ & 98 & DMSO & $\mathbf{T}$ & $123-01-3$ & - & $-(34)$ \\
\hline 14. & 2-Ethyl-1-hexanol & $\begin{array}{c}\mathrm{CH}_{8}\left(\mathrm{CH}_{2}\right)_{8} \mathrm{CH}\left(\mathrm{C}_{2} \mathrm{H}_{5}\right)- \\
\mathrm{CH}_{2} \mathrm{OH}\end{array}$ & 98 & DMSO & W & $104-76-7$ & - & $?$ \\
\hline 15. & Ethylenebisoctadecanamide & $\begin{array}{c}\mathrm{C}_{17} \mathrm{H}_{85} \mathrm{CONHCH}_{2} \mathrm{CH}_{2-} \\
\mathrm{HNOCH}_{35} \mathrm{C}_{17}\end{array}$ & 99 & DMSO & $\mathbf{T}$ & $110-30-5$ & - & $?$ \\
\hline 16. & 2-Ethoxyethanol & $\mathrm{C}_{2} \mathrm{H}_{5} \mathrm{OC}_{2} \mathrm{H}_{4} \mathrm{OH}$ & 98 & DMSO & W & $110-80-5$ & - & $-(7)$ \\
\hline 17. & Glycerol & $\mathrm{C}_{3} \mathrm{H}_{5}(\mathrm{OH})_{3}$ & 99.6 & $\mathrm{H}_{2} \mathrm{O}$ & W & $56-81-5$ & - & $-(7,9)$ \\
\hline 18. & Hexafluorosilicic acid & $\mathbf{H}_{2} \mathrm{SiF}_{6}$ & $40-45$ & $\mathrm{H}_{2} \mathrm{O}$ & W & $16961-83-4$ & - & $?$ \\
\hline 19. & Hexamethylene tetramine & $\left(\mathrm{CH}_{2}\right)_{6} \mathrm{~N}_{4}$ & 99.9 & $\mathrm{H}_{2} \mathrm{O}$ & W & $100-97-0$ & + & $+(5-9)$ \\
\hline 20. & Hexanedioic acid & $\mathrm{HOOC}\left(\mathrm{CH}_{2}\right)_{4} \mathrm{COOH}$ & 99 & DMSO & $\mathbf{T}$ & $124-04-9$ & - & $?$ \\
\hline 21. 3 & $\begin{array}{l}\text { 3-Hydroxy-2-naphthoic } \\
\text { acid }\end{array}$ & $\mathrm{HOC}_{10} \mathrm{COOH}$ & 99.9 & DMSO & $\mathbf{T}$ & $92-70-6$ & - & $?$ \\
\hline 22. & Iron(III)chloride & $\mathrm{FeCl}_{3}$ & 99 & $\mathrm{H}_{2} \mathrm{O}$ & W & $7705-08-0$ & - & $-(7,36)$ \\
\hline 23. & Isobutyl alcohol & $\mathrm{C}_{4} \mathrm{H}_{9} \mathrm{OH}$ & 99.7 & $\mathrm{H}_{2} \mathrm{O}$ & W & $78-83-1$ & - & $+(14,15)$ \\
\hline 24. & Methanol & $\mathrm{CH}_{3} \mathrm{OH}$ & 99.6 & $\mathrm{H}_{2} \mathrm{O}$ & W & $67-56-1$ & - & $-(7,24)$ \\
\hline 25. & Methyl acetoacetate & $\mathrm{CH}_{3} \mathrm{COCH}_{2} \mathrm{COOCH}_{3}$ & 99 & $\mathrm{H}_{2} \mathrm{O}$ & $\mathbf{T}$ & $105-45-3$ & - & $?$ \\
\hline 26. & $\begin{array}{l}\text { 4,4'-Methylenedi- } \\
\text { phenyldiisocyanate }\end{array}$ & $\mathrm{CH}_{2}\left(\mathrm{C}_{6} \mathrm{H}_{4} \mathrm{NCO}\right)_{2}$ & 98 & DMSO & $\mathbf{T}$ & $101-68-8$ & + & $?(12)$ \\
\hline 27. & 4-Methyl-2-pentanol & $\begin{array}{c}\left(\mathrm{CH}_{8}\right)_{2} \mathrm{CHCH}_{2} \mathrm{CH}- \\
(\mathrm{OH}) \mathrm{CH}_{8}\end{array}$ & 98 & DMSO & $\mathbf{T}$ & $108-11-2$ & - & $?$ \\
\hline 28. 1 & $p$-Nonylphenol & $\mathrm{C}_{6} \mathrm{H}_{4}\left(\mathrm{C}_{9} \mathrm{H}_{19}\right)(\mathrm{OH})$ & 89 & DMSO & $\mathbf{T}$ & $104-40-5$ & - & $?$ \\
\hline 29. & Octadecanoic acid & $\mathrm{CH}_{3}\left(\mathrm{CH}_{2}\right)_{16} \mathrm{COOH}$ & 60 & Acetone & W & $57-11-4$ & - & $\begin{aligned}-(7,9 \\
15,31)\end{aligned}$ \\
\hline 30. & Oleic acid & $\mathrm{C}_{17} \mathrm{H}_{38} \mathrm{COOH}$ & 99.9 & DMSO & W & $112-80-1$ & - & $\begin{array}{c}-(7,9 \\
15,30 \\
31)\end{array}$ \\
\hline 31. 1 & Pentaerythritol & $\mathrm{C}\left(\mathrm{CH}_{2} \mathrm{OH}\right)_{4}$ & 90 & $\mathrm{H}_{2} \mathrm{O}$ & $\mathbf{W}$ & $115-77-5$ & - & $?$ \\
\hline & Pentasodium triphosphate & $\mathrm{Na}_{5} \mathbf{P}_{\mathbf{8}} \mathrm{O}_{10}$ & 90 & $\mathrm{H}_{2} \mathrm{O}$ & W & $13573-18-7$ & - & $?$ \\
\hline 33. 2 & 2-Propanol & $\mathrm{CH}_{3} \mathrm{CH}(\mathrm{OH}) \mathrm{CH}_{3}$ & 99 & $\mathrm{H}_{2} \mathrm{O}$ & W & $67-63-0$ & - & $+(15-17)$ \\
\hline 34. & Sodium dithionite & $\mathrm{Na}_{2} \mathrm{~S}_{2} \mathrm{O}_{4}$ & 89.1 & $\mathrm{H}_{2} \mathrm{O}$ & W & $7775-14-6$ & - & $?$ \\
\hline 35. 7 & Tetraffuoroboric acid & $\mathrm{HBF}_{4}$ & 42 & $\mathrm{H}_{2} \mathrm{O}$ & W & $16872-11-0$ & - & $?$ \\
\hline 36. 7 & Tetramethylenesulfone & $\mathrm{C}_{4} \mathrm{H}_{8} \mathrm{SO}_{2}$ & 99 & $\mathrm{H}_{2} \mathrm{O}$ & $\mathbf{T}$ & $126-33-0$ & - & $?$ \\
\hline 37. 7 & Trichloroisocyanuric acid & $\mathrm{C}_{3} \mathrm{Cl}_{8} \mathrm{~N}_{3} \mathrm{O}_{3}$ & 97 & $\mathrm{H}_{2} \mathrm{O}$ & $\mathbf{T}$ & $87-90-1$ & - & $?$ \\
\hline 38. & Urea & $\mathrm{H}_{2} \mathrm{NCONH}_{2}$ & 99 & $\mathrm{H}_{2} \mathrm{O}$ & W & $57-13-6$ & - & $-(20)$ \\
\hline 39. $p$ & p-Xylene & $\mathrm{C}_{6} \mathrm{H}_{4}\left(\mathrm{CH}_{3}\right)_{2}$ & 98 & DMSO & $\mathbf{W}$ & $106-42-3$ & - & $?$ \\
\hline
\end{tabular}

T: Tokyo Kasei Kogyo Co. LTD. Tokyo, Japan. W: Wako Pure Chemical Industries, LTD., Osaka, Japan. 
Table 2. List of 4 volatile compounds tested for mutagenicity.

\begin{tabular}{cllcccccc}
\hline No. & Name (IUPAC) & Structure & $\begin{array}{c}\text { Purity } \\
(\%)\end{array}$ & Diluent & Source & CAS No. & $\begin{array}{c}\text { Mutage- Carcino- } \\
\text { nicity }\end{array}$ \\
genicity
\end{tabular}

TAK: Takachiho Chemical Co. Tokyo, Japan.

pounds, excluding four volatile ones (ammonia, butane, chlorine and 2-methylpropene) was tested by the preincubation method ${ }^{4}$ with $\mathrm{S} 9 \mathrm{mix}$, which was slightly modified from the Ames test." When E. coli instead of $S$. typhimurium was used, histidine and biotin in the top agar were replaced by tryptophan at the same concentration. The 39 compounds tested were dissolved in sterilized diluents, as shown in Table 1, and $0.1 \mathrm{ml}$ of bacterial strain and $0.5 \mathrm{ml}$ of $\mathrm{S} 9 \mathrm{mix}$ or sodium phosphate buffer (pH 7.4) were added to a sterile test tube containing $0.1 \mathrm{ml}$ of various concentrations of the chemicals. This mixture was preincubated in a shaker water bath at $37^{\circ} \mathrm{C}$ for 20 minutes, and then added to $2 \mathrm{ml}$ of molten top agar $\left(45^{\circ} \mathrm{C}\right)$. The contents of each tube were mixed and poured onto a minimal glucose agar plate immediately. The plates were incubated at $37^{\circ} \mathrm{C}$ for 48 hours, and then the number of revertant colonies on each plate was scored with an automated colony counter.

In the test of such gaseous or highly volatile compounds as ammonia, butane, chlorine and 2-methylpropane, a minimal glucose agar plate covered with $0.1 \mathrm{ml}$ of one of the strains and 0.5 $\mathrm{ml}$ of $\mathbf{S 9} \mathrm{mix}$ or buffer was placed upside down without a lid inside a glass chamber. This method has been described in detail elsewhere. ${ }^{5)}$

All these tests were performed in duplicate. 2-(2-Furyl)-3-(5-nitro-2-furyl) acrylamide (AF-2), $\mathrm{N}$-ethyl-N'-nitro- $\mathrm{N}$-nitrosoguanidine (ENNG), 9-aminoacridine (9AC), 4-nitroquinoline-1-oxide (4NQO), benzo(a)pyrene (B(a)P), 2-aminoanthracene (2AA) and 2-nitrofluorene (12NF) were used as positive control (see in Table 4).

A contamination test was carried out in each experiment. The background bacterial lawn was checked routinely by dissected microscope.

\section{RESULTS AND DISCUSSION}

The results of the mutagenicity of the 39 tested compounds, which were in crystal, powdered or liquid form, are shown in Table 1 and those of four highly volatile or gaseous compounds in Table 2. The details with regard to the mutagenicity of each compound are given in Tables 4 and 5 .

Seven compounds-1-dodecane (DOD), chlorine (CH), oleic acid (OLE), 2-ethyl-1-hexanol (2HE), $p$-nonylphenol (NP), trichloroisocyanuric acid (TCI) and $p$-xylene (XY)-were toxic to the bacterial strains used for the test. DOD, CH and OLE were toxic at $50 \mu \mathrm{g} /$ plate to $S$. typhimurium, and $2 \mathrm{HE}, \mathrm{NP}, \mathrm{TCI}$ and $\mathrm{XY}$ at 50 or $100 \mu \mathrm{g} / \mathrm{plate}$ to both $S$. typhimurium and $E$. coli.

Only two out of the 43 compounds-hexamethylentetramine (HMT) and 4,4'-methylenediphenyldi$i_{\text {socyanate (MDI)-were mutagenic. The other }}$ 41 compounds presented no mutagenic activity either in the presence or absence of $\mathbf{S} 9$ mix. The mutagenicity of 11 out of the 43 compounds $(25.6 \%)$ has already been reported by mutation tests including chromosomal aberration, sister chromatid exchange and dominant lethal tests. Thirty-two out of the 43 compounds $(74.4 \%)$ had not been tested, nor have their mutagenicity been reported. Only 14 out of the 43 compounds $(32.5 \%)$ have been tested for their carcinogenicity; carcinogenicity was detected in two chemicals and one manufacturing process of a chemical.

HMT was mutagenic to $S$. typhimurium TA100 with $\mathbf{S 9} \mathrm{mix}$ and to TA98 with and without $\mathrm{S} 9$ mix. There was a weak dose-response correlation between the number of histidine revertant colonies (His ${ }^{+}$) of TA100 and the concentration of the chemical in the absence of $\mathbf{S 9}$ mix. The mutagenicity of the chemical has not been reported so far. 
Table 3. The annual output in Japan of the 43 chemicals tested for mutagenicity $(1,000 \mathrm{t} / \mathrm{year})$.

\begin{tabular}{|c|c|c|c|c|c|c|}
\hline No. & Chemicals (IUPAC) & 1979 & 1981 & 1982 & 1983 & 1984 \\
\hline 1. & Aluminium fluoride & 61 & 61 & & 45 & 45 \\
\hline 2. & $\begin{array}{l}\text { 3-Amino-6-chlorotoluene- } \\
\text { 4-sulfonic acid }\end{array}$ & 1 & 1 & & 1 & 1 \\
\hline 3. & 1-Amino-2-propanol & 1 & 1 & & & 1 \\
\hline 4. & Ammonium fluoride & 3 & 3 & & & 3 \\
\hline 5. & Ammonium peroxodisulfate & 4 & 4 & & 3 & 3 \\
\hline 6. & Barium chloride & 10 & & 9 & 9 & 11 \\
\hline 7. & 2-Butanone & 88 & & 97 & 102 & 111 \\
\hline 8. & Butyl acetate & 36 & & 29 & 31 & 34 \\
\hline 9. & Cerium(IV)dioxide & 2 & 2 & & 2 & 2 \\
\hline 10. & Copper(I)oxide & 4 & 4 & & 6 & 4 \\
\hline 11. & Decanedioic acid & 2 & 2 & & 2 & 1 \\
\hline 12. & 1-Dodecanol & 78 & & 76 & & \\
\hline 13. & Dodecylbenzene & 200 & & 157 & 180 & \\
\hline 14. & 2-Ethyl-1-hexanol & 264 & & 226 & 219 & \\
\hline 15. & Ethylenebisoctadecanamide & 4 & 4 & & 4 & 1 \\
\hline 16. & 2-Ethoxyethanol & 41 & & 50 & 54 & 61 \\
\hline 17. & Glycerol & 70 & & 77 & 72 & 71 \\
\hline 18. & Hexafluorosilicic acid & 2 & 2 & & 2 & 2 \\
\hline 19. & Hexamethylenetetramine & 21 & & 13 & 15 & 16 \\
\hline 20. & Hexanedioic acid & 47 & 47 & & 47 & 47 \\
\hline 21. & 3-Hydroxy-2-naphthoic acid & 3 & 3 & & 2 & 1 \\
\hline 22. & Iron(III)chloride & 211 & & 247 & 252 & 281 \\
\hline 23. & Isobutyl alcohol & 49 & & 33 & 29 & 26 \\
\hline 24. & Methanol & 940 & & 625 & 373 & 280 \\
\hline 25. & Methyl acetoacetate & 3 & 3 & & 3 & 3 \\
\hline 26. & 4,4'-Methylenediphenyldiisocyanate & 44 & & 58 & 70 & 85 \\
\hline 27. & 4-Methyl-2-pentanol & 3 & 3 & 3 & & 3 \\
\hline 28. & p-Nonylphenol & 16 & 16 & 16 & & 16 \\
\hline 29. & Octadecanoic acid & 26 & & 23 & 8 & 8 \\
\hline 30. & Oleic acid & 10 & & 16 & 15 & 16 \\
\hline 31. & Pentaerythritol & 40 & & 31 & 32 & 30 \\
\hline 32. & Pentasodium triphosphate & 122 & & 79 & 66 & 61 \\
\hline 33. & 2-Propanol & 96 & 1 & 96 & 90 & 92 \\
\hline 34. & Sodium dithionite & 31 & & 35 & 33 & 34 \\
\hline 35. & Tetrafluoroboric acid & 1 & 1 & & 1 & 1 \\
\hline 36. & Tetramethylenesulfone & 1 & 1 & & & \\
\hline 37. & Trichloroisocyanuric acid & 10 & 10 & & 10 & 10 \\
\hline 38. & Urea & 2,083 & & 1,185 & 981 & 1,120 \\
\hline 39. & p-Xylene & 647 & & 583 & 620 & 683 \\
\hline 1. & Ammonia & 2,831 & & 2,010 & 2,010 & 2,029 \\
\hline $\begin{array}{l}2 . \\
3 .\end{array}$ & $\left.\begin{array}{l}\text { Butane } \\
\text { 2-Methylpropene(Butylene) }\end{array}\right\}$ & 1,745 & & 1,466 & 1,434 & 1,526 \\
\hline 4. & Chlorine & 2,700 & & 2,471 & 3,265 & 2,748 \\
\hline
\end{tabular}

IUPAC: International Union of Pure and Applied Chemistry. 


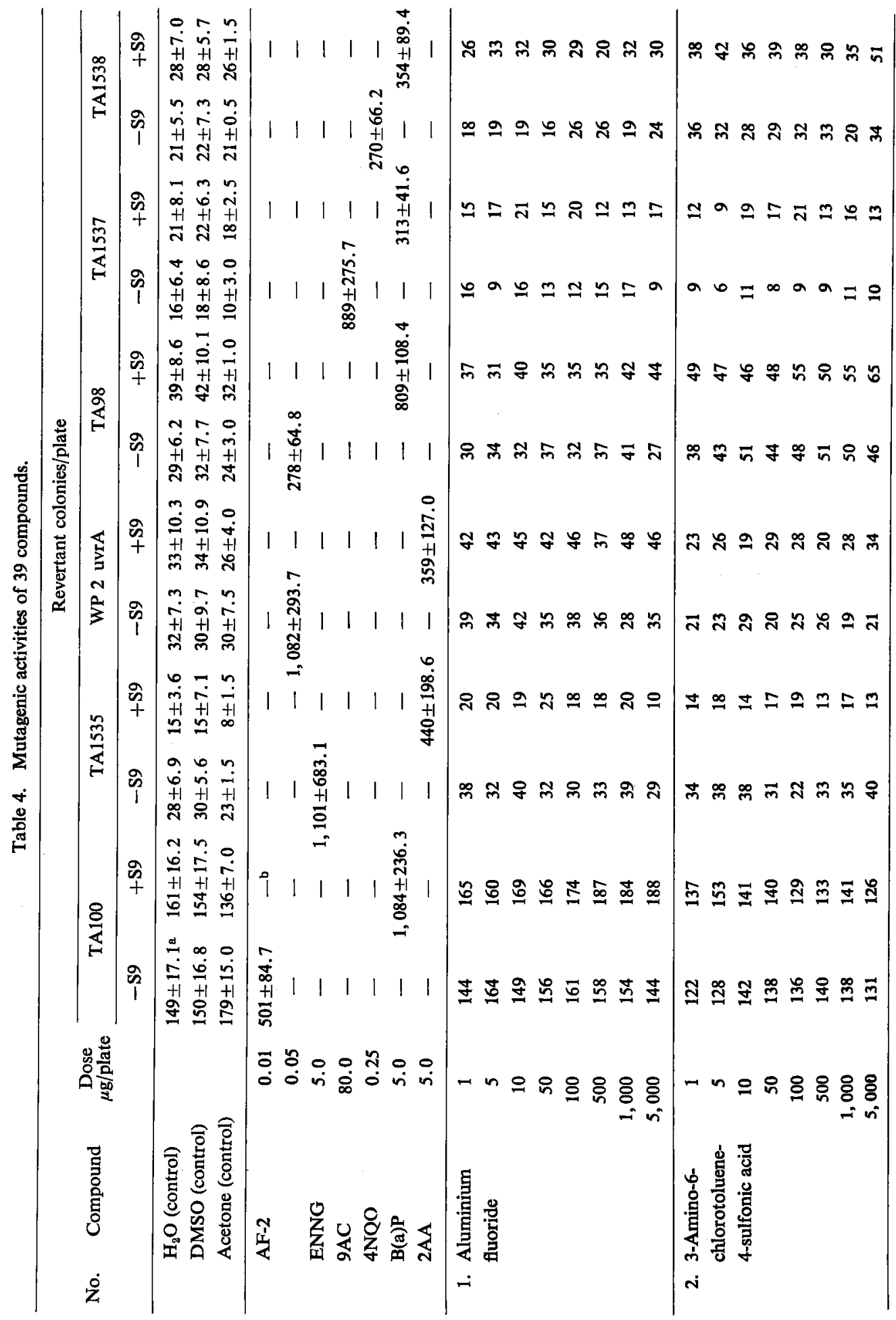




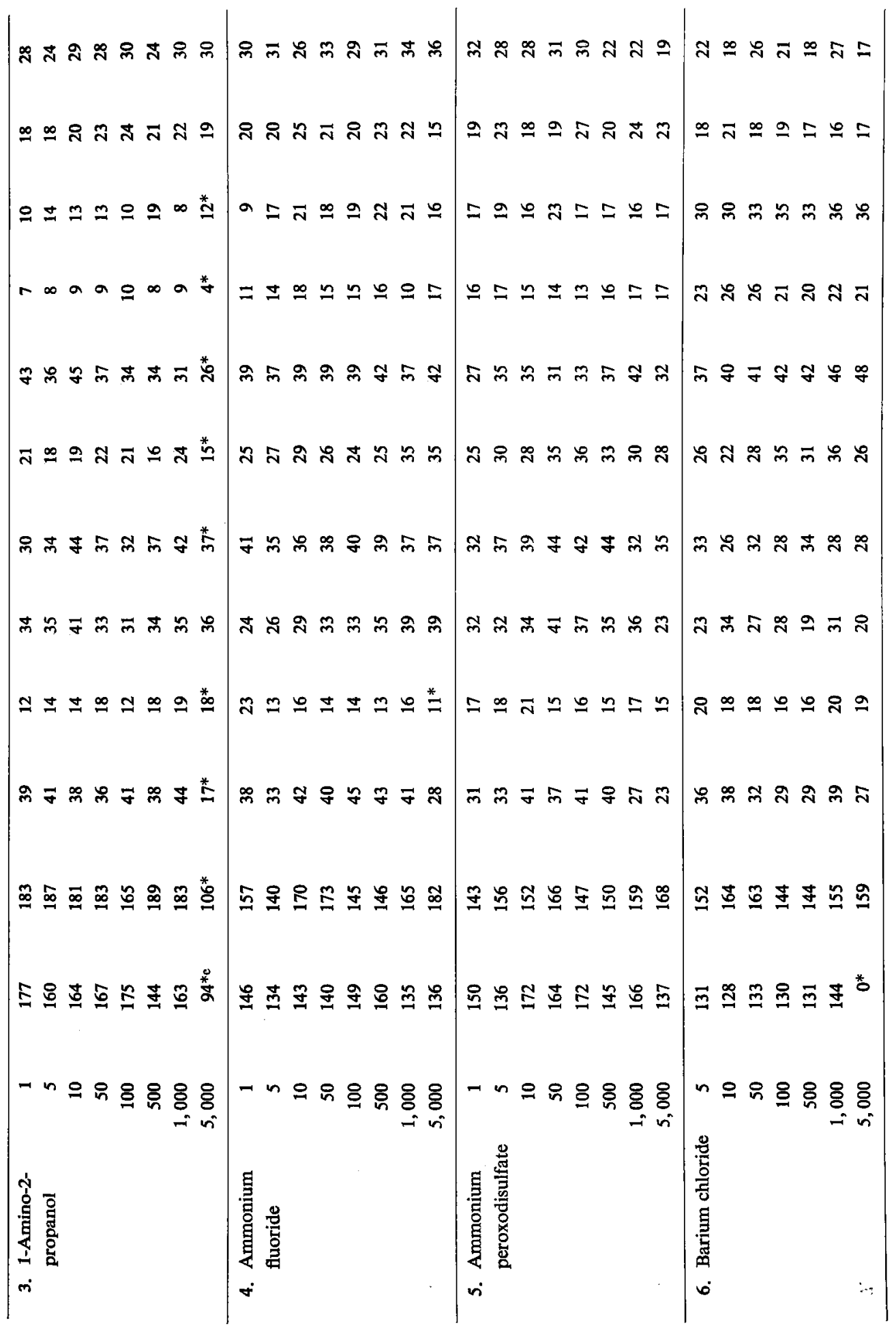




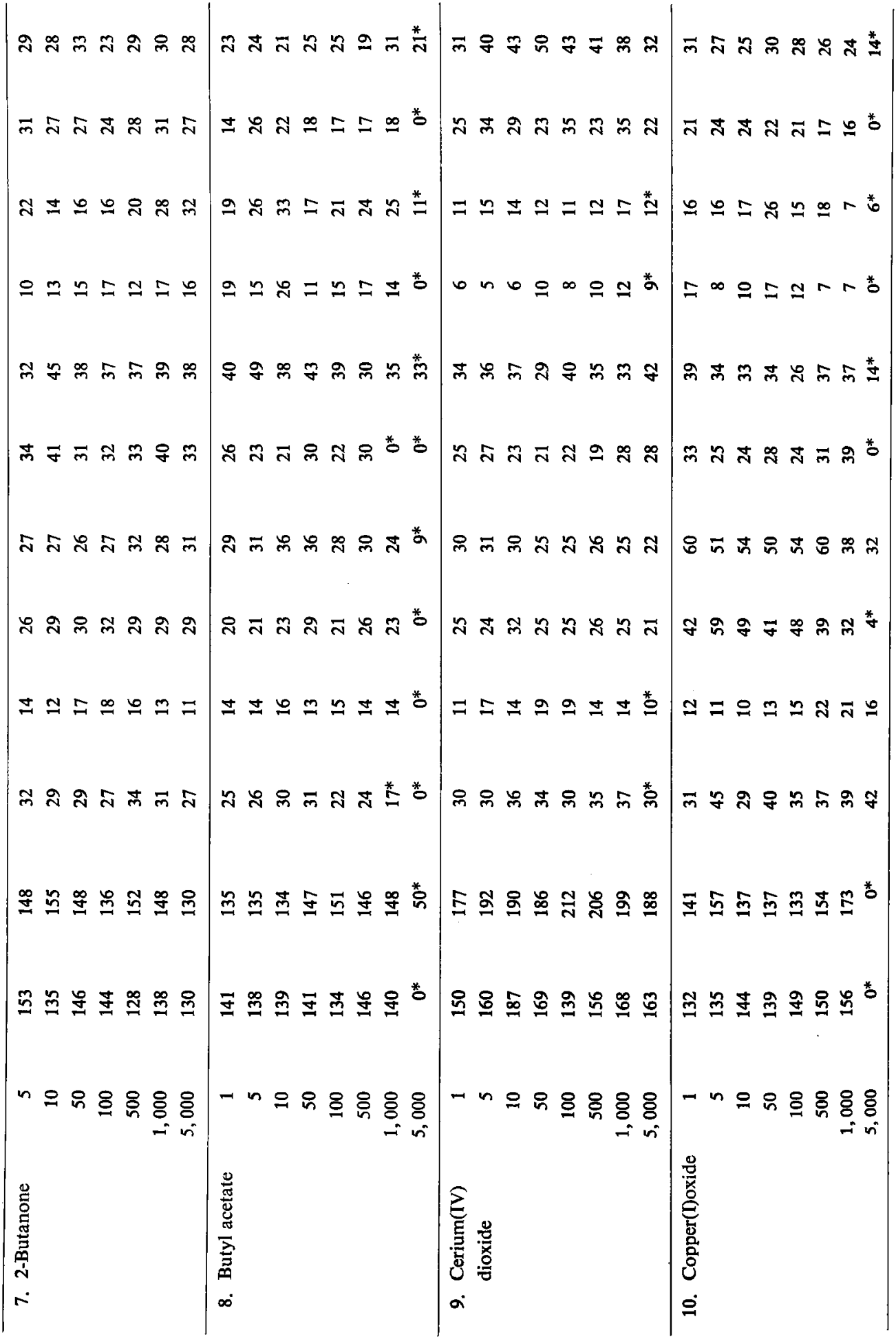




\begin{tabular}{|c|c|c|c|}
\hline 蛋 & 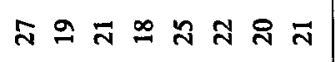 & 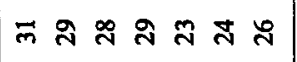 & 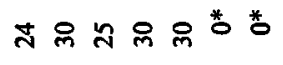 \\
\hline 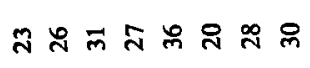 & 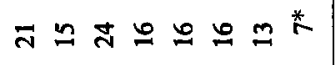 & $\because$ ニ & ๗ \\
\hline กัมูป & สุ & 䑻 & 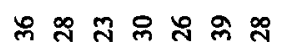 \\
\hline$I m \pm \approx m=\infty \approx$ & 음ำ & 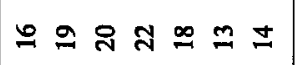 & $m=\simeq \pm \simeq \simeq$ \\
\hline 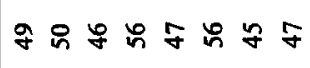 & 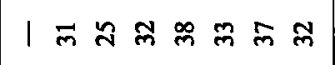 & 品 న్లు స స & 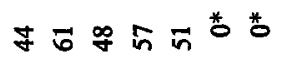 \\
\hline 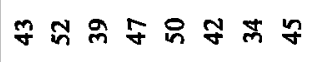 & 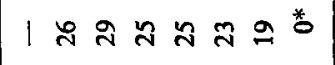 & ส & 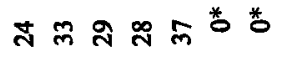 \\
\hline "กส & | \& 寸 & 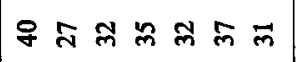 & 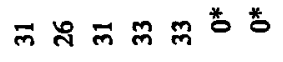 \\
\hline 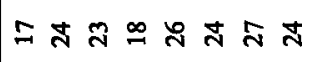 & 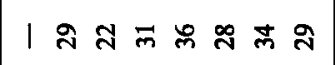 & ป & 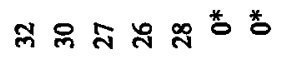 \\
\hline$a=\simeq 20 \Omega \simeq \pm$ & 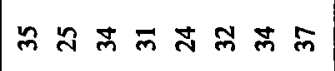 & 9 & $\approx \Omega \stackrel{2}{2} \underbrace{*}$ * \\
\hline 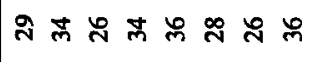 & m & 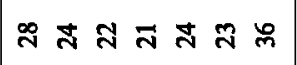 & 윢 ํㅜ m \\
\hline 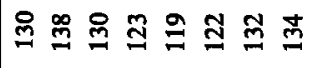 & 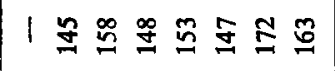 & 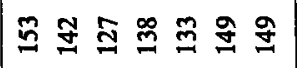 & 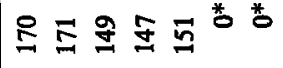 \\
\hline 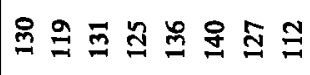 & | & 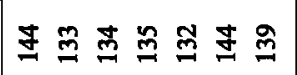 & 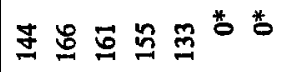 \\
\hline -n= & 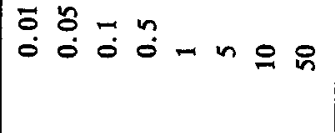 & n. & -n 으용용 \\
\hline 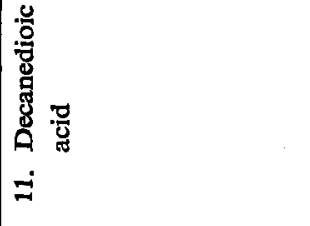 & 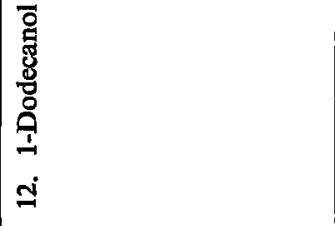 & 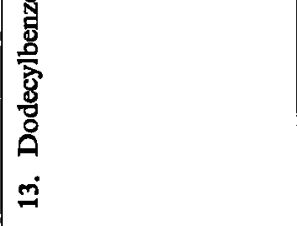 & 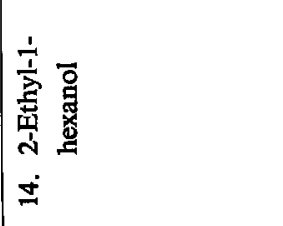 \\
\hline
\end{tabular}




\begin{tabular}{|c|c|c|c|}
\hline 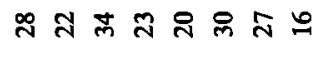 & 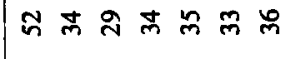 & : & 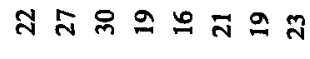 \\
\hline 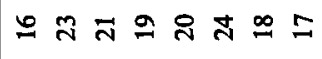 & 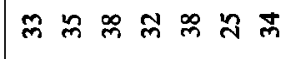 & 는 & $\cong \simeq \cong \approx \stackrel{\infty}{\sim}$ 요 \\
\hline$\simeq \cong=\simeq \infty \cong \simeq$ 은 & 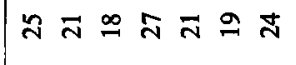 & ชิ & สิ ป ส \\
\hline 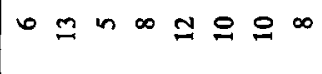 & $\simeq \pm உ \cong \Xi \Xi \simeq$ & $\simeq 9=n+\simeq 2$ & 옴프. \\
\hline 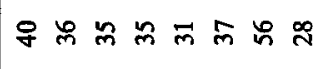 & 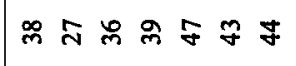 & 舟 웅 & 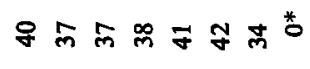 \\
\hline$\stackrel{\sim}{N} \bar{m} \tilde{\sim} \tilde{m}$ & స足 & 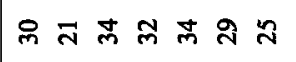 & 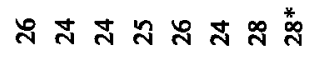 \\
\hline nీ ₹ & 시요 $\infty$ & 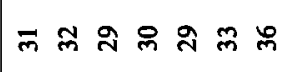 & 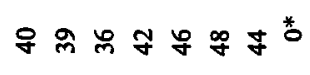 \\
\hline$\tilde{N} m g \bar{m} \bar{m} N \stackrel{N}{N}$ & 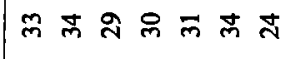 & 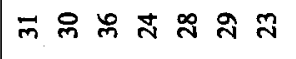 & 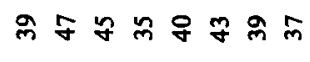 \\
\hline I늘 & 우응ㅇㅇㅇㅛ & $\pm 2 \cong=\Xi \cong \pm$ & 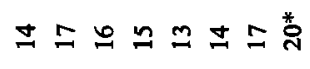 \\
\hline డ సి $\approx$ న్ల & 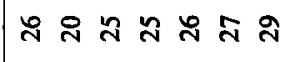 & 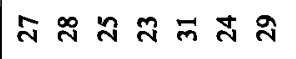 & $\bar{m} \tilde{m} \tilde{m} \tilde{m} m \tilde{m}$ \\
\hline 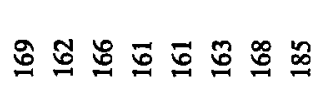 & 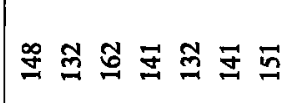 & 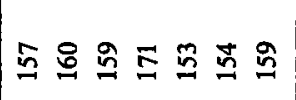 & 똥 \\
\hline 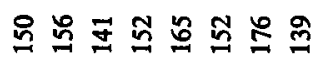 & 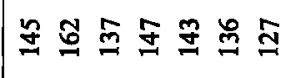 & 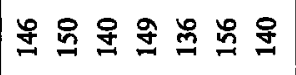 & 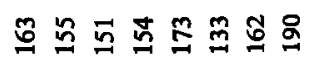 \\
\hline 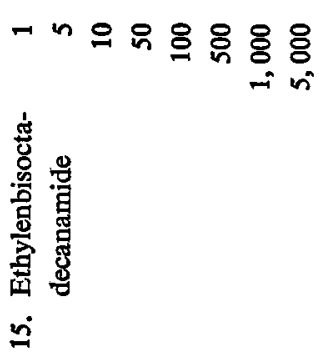 & 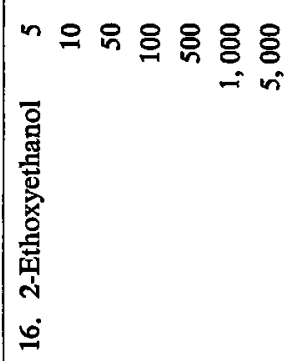 & nᄋ요용ㅇㅇㅇㅇ & 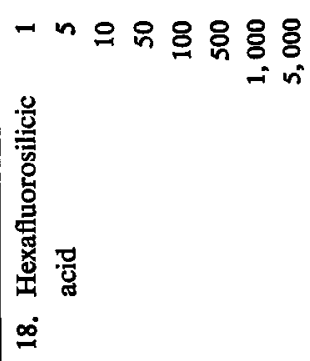 \\
\hline
\end{tabular}




\begin{tabular}{|c|c|c|c|}
\hline 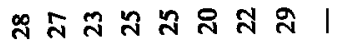 & 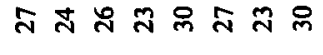 & $\tilde{m} \bar{m} \bar{m} \tilde{m} \bar{q} \tilde{N}$ & $\bar{N} \mathscr{N} \bar{N} \tilde{N} \mathscr{N} \mathfrak{N}$ \\
\hline 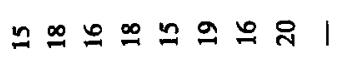 & $\simeq \stackrel{\sim}{\sim}$ & 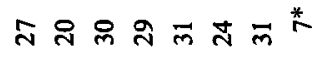 & $\simeq \Xi \pm \pm \cong \cong=$ \\
\hline 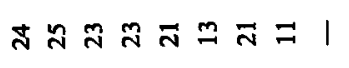 & $\cong$ 유 $\cong \mathscr{\sim} \cong \simeq$ & 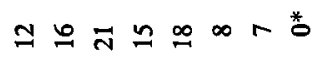 & ㄱํำ \\
\hline$\Rightarrow n n \infty=a$ 이 & $\Rightarrow a$ o a bor & 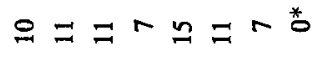 & 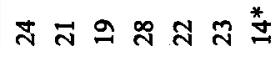 \\
\hline 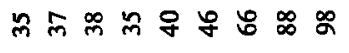 & 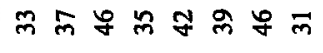 & 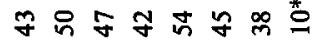 & 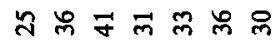 \\
\hline 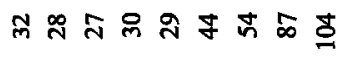 & సి & 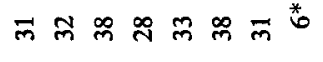 & 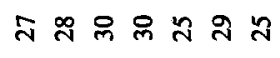 \\
\hline 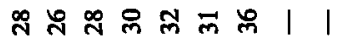 & 央 品 & กัก ั & $\hbar$ స \\
\hline m సి & 趈 \& & 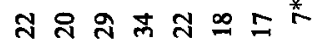 & 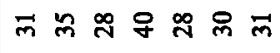 \\
\hline $\mathscr{\simeq} \pm \simeq \pm \simeq \simeq \simeq 1$ & $\because$ $\cong$ ล & $\therefore=\cong \simeq \simeq \simeq a \infty$ & 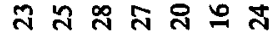 \\
\hline ส & 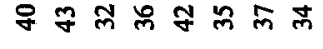 & 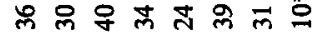 & 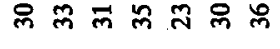 \\
\hline 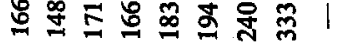 & 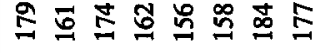 & 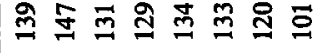 & 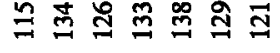 \\
\hline 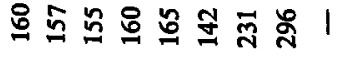 & 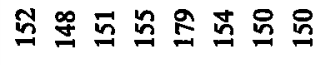 & 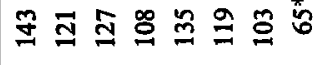 & 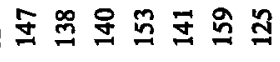 \\
\hline 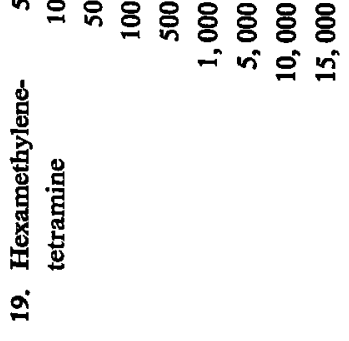 & 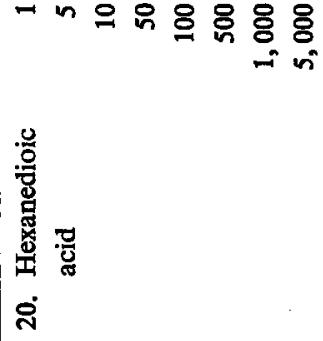 & 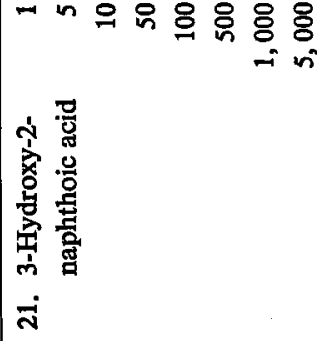 & 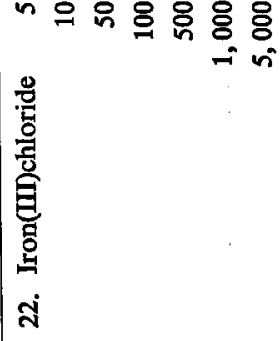 \\
\hline
\end{tabular}




\begin{tabular}{|c|c|c|c|}
\hline 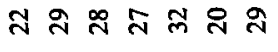 & 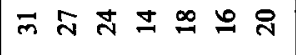 & $\tilde{N}$ సे & 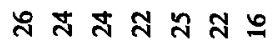 \\
\hline$=\check{N} \vec{N} \vec{N} \tilde{N}$ & 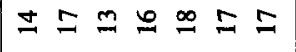 & స ర్ల & 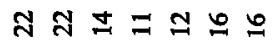 \\
\hline స్ల & 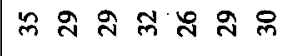 & $\bar{N}$ ลิ 2 Lั นัN & $=\mathscr{N} \vec{N} \approx \tilde{N}$ \\
\hline 뮤 䒬 & 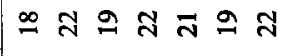 & $\Xi \mathscr{\pm} \pm \pm \infty \cong n$ & 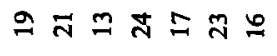 \\
\hline 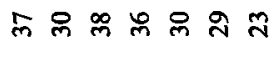 & 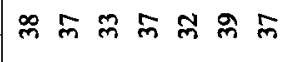 & 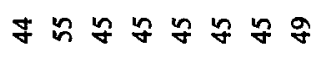 & 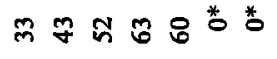 \\
\hline 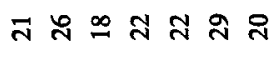 & 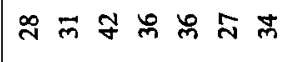 & $\tilde{m}$ & 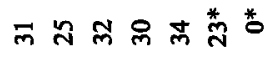 \\
\hline $\bar{m}$ ర్d సి & 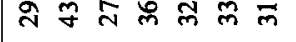 & 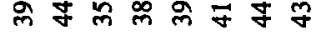 & 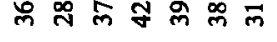 \\
\hline$\vec{m}$ ని & 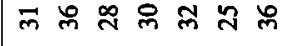 & 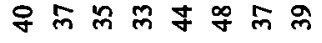 & 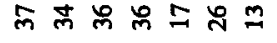 \\
\hline 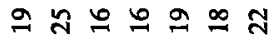 & $\div r=m ㅇ=$ & $\stackrel{\infty}{2} \stackrel{\circ}{=}=\infty$ 유 & $\Rightarrow \cong=\vec{n}=\cong n$ \\
\hline 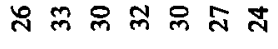 & สㅇำ & 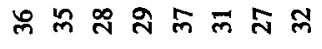 & ホ \\
\hline 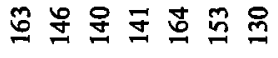 & 車志怘 & 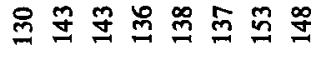 & 응 \\
\hline 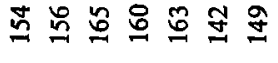 & 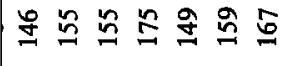 & 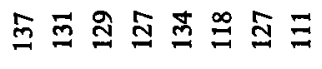 & 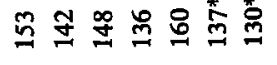 \\
\hline 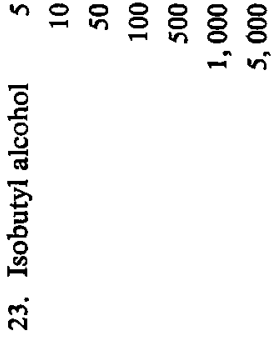 & 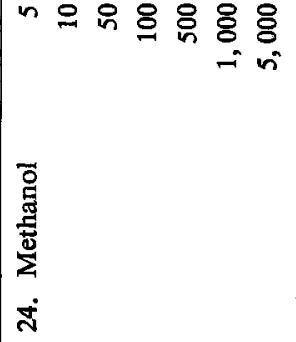 & 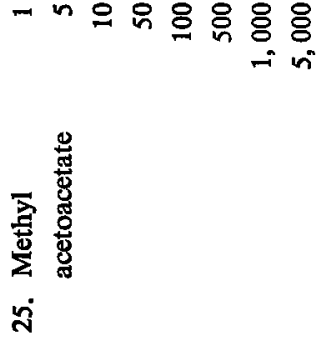 & 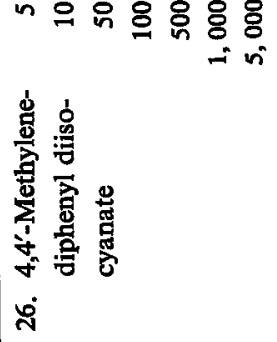 \\
\hline
\end{tabular}




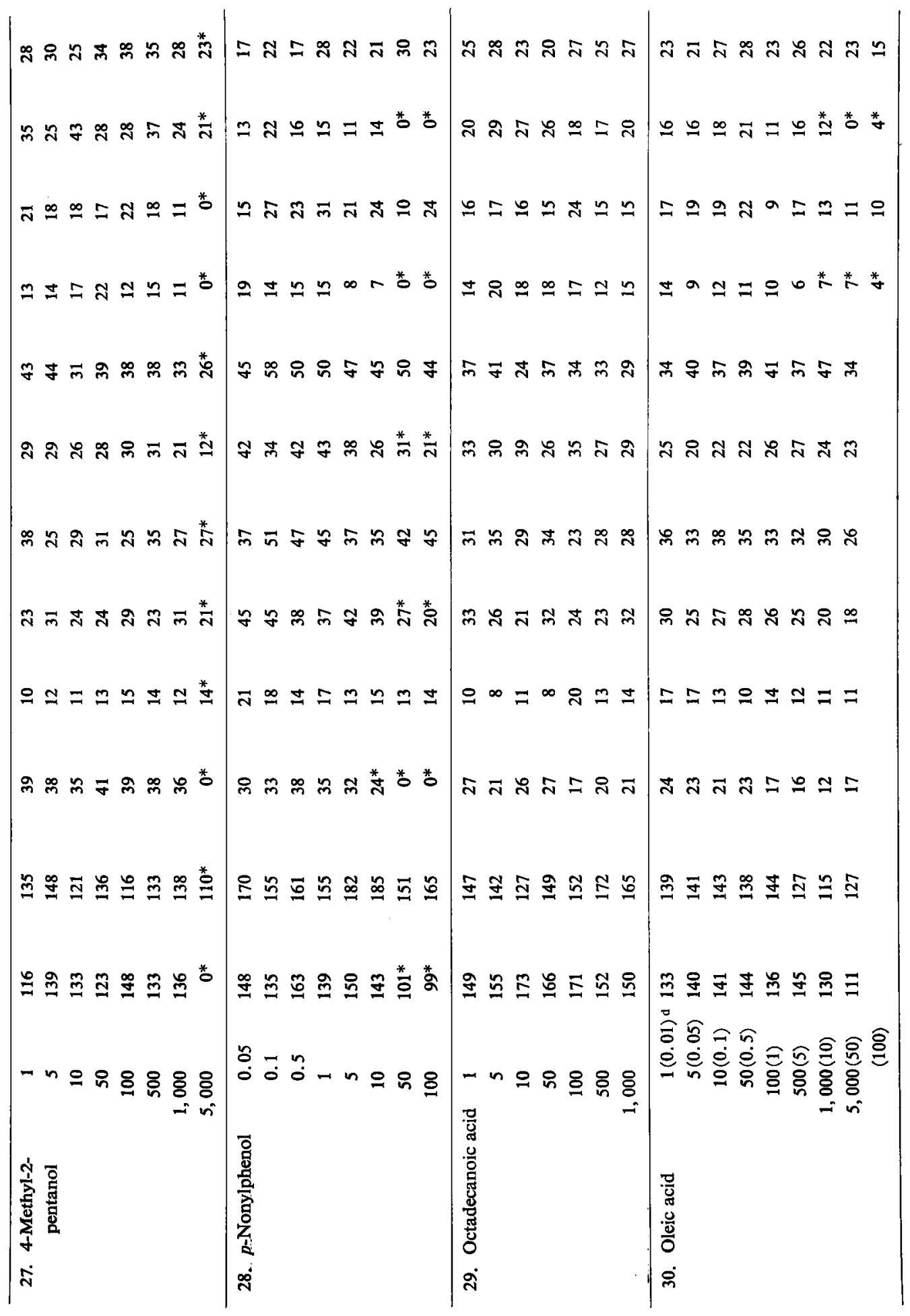




\begin{tabular}{|c|c|c|c|}
\hline 고 2 స & 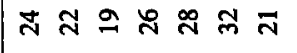 & 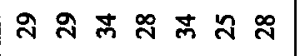 & 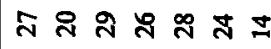 \\
\hline$m \pm \mathscr{N}$ & สักักทัง & กิ & $\simeq \approx \cong \mathscr{\sim} \cong \simeq$ \\
\hline 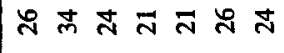 & $\ddot{\sim} \bar{m} \dot{n}=\stackrel{\infty}{\sim}$ & ส & $\bar{\sim} \tilde{m} \bar{N} \stackrel{\sim}{\sim} \stackrel{\sim}{\sim}$ \\
\hline 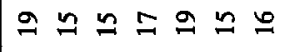 & $=$ 응 & $\pm \Xi \pm 0 \cong \pm \infty$ & 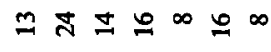 \\
\hline 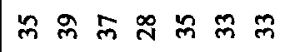 & 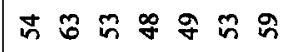 & F & 足 \\
\hline Nิ & 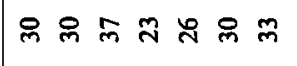 & 舫 & 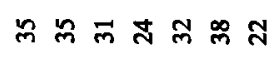 \\
\hline 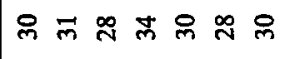 & 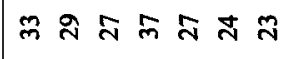 & స్ల ర్లి ర్ల స సి & $\infty$ \\
\hline 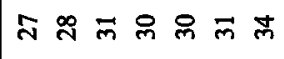 & స స & సิ & $\bar{\pi} \tilde{m} \approx \pm \delta \bar{N}$ \\
\hline$\beth$ สิ $\cong \pm \cong \cong \pm$ & 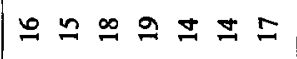 & 의음의 & 구 $\vec{N} \cong \simeq \simeq$ \\
\hline 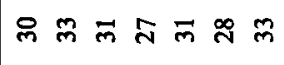 & సి & 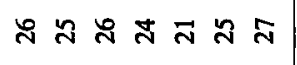 & 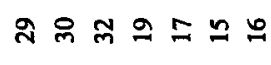 \\
\hline 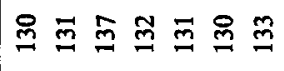 & 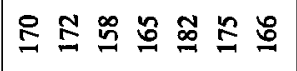 & 禹号怘怘怘 & 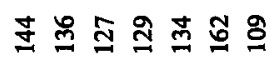 \\
\hline 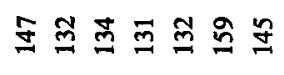 & 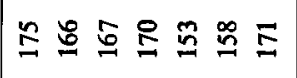 & 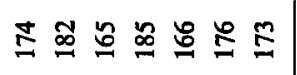 & 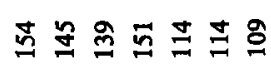 \\
\hline 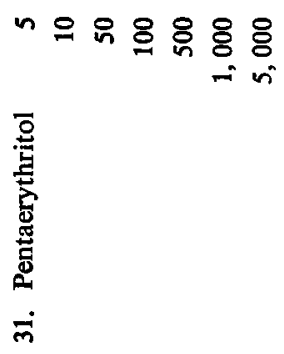 & 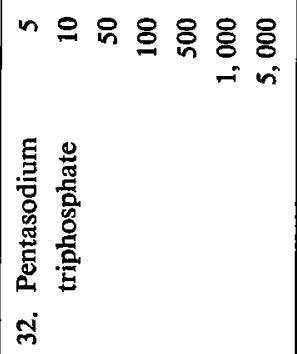 & 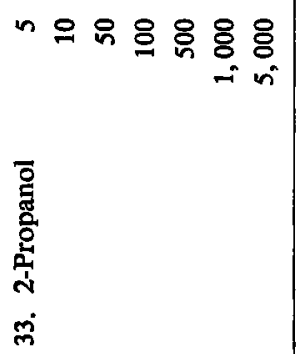 & n 응요 \\
\hline
\end{tabular}




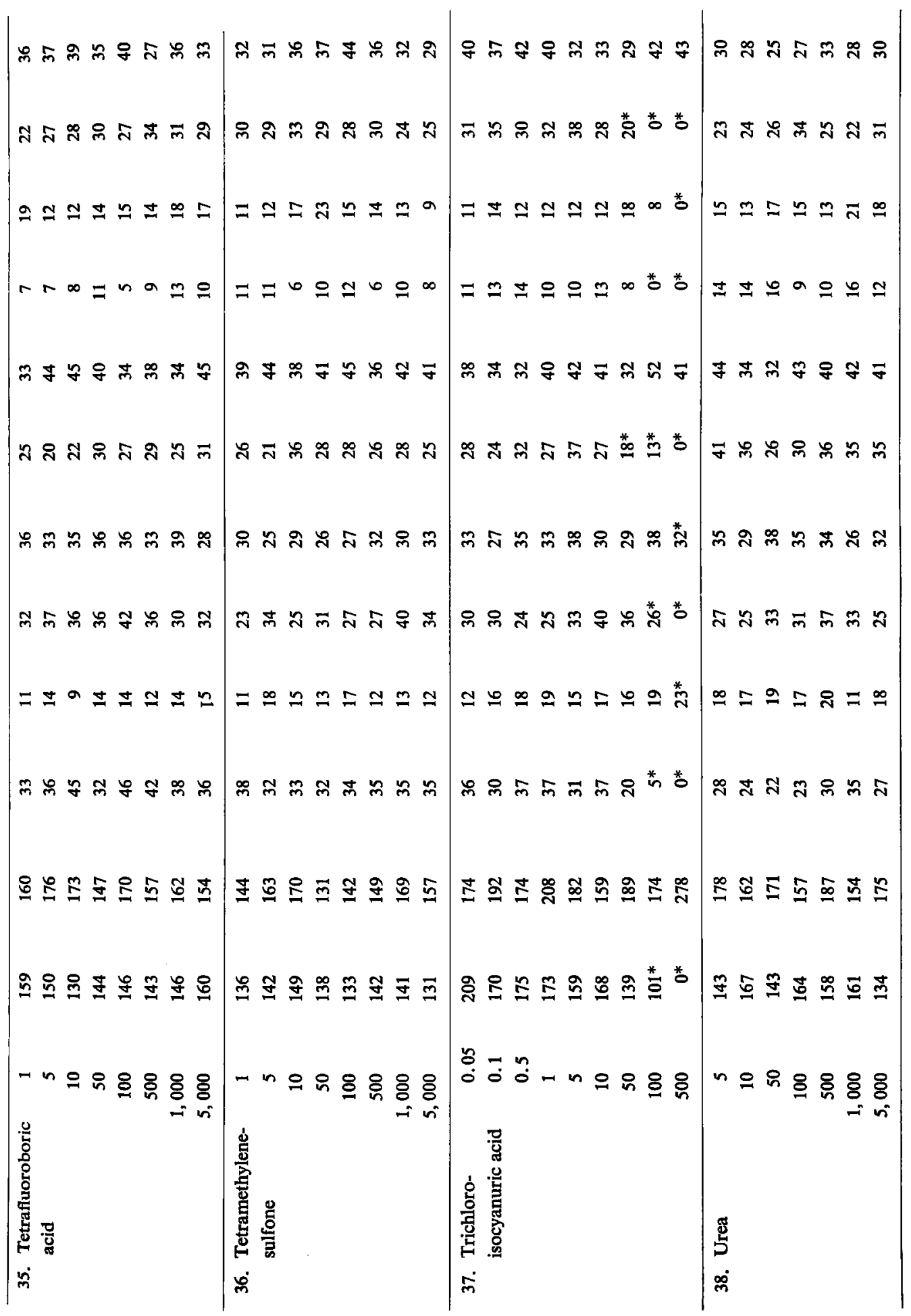


The dominant lethal mutation in $\mathrm{C} 3 \mathrm{H}$ male mice given the substance orally at a dose of $25,000 \mathrm{mg} / \mathrm{kg}$ body weight was reported to be positive but negative at $800-1,500 \mathrm{mg} / \mathrm{kg}$ body weight when administered intraperitoneally. ${ }^{\text {) }}$ ( Mammary and pituitary tumors were induced in rats given drinking water containing $0.1 \% \mathrm{HMT},{ }^{\text {") }}$ and multiple malignant tumors were observed in rats given HMT subcutaneously, ${ }^{\text {s) }}$ but not in mice.9) On the other hand, there are negative reports on rats and mice given drinking water containing HMT. ${ }^{10)}$ No malformation was observed in rats given drinking water containing $1 \%$ HMT for two weeks before mating or during pregnancy and lactation.11) The output of this chemical in Japan was 21 million kilograms in 1979 and 13 million kilograms in 1982. ${ }^{121}$

MDI was mutagenic to $S$. typhimurium TA100 and slightly mutagenic to TA98 by metabolic activation. These results were in agreement with those reported previously. ${ }^{13,14)}$ This compound gave positive results at $100 \mu \mathrm{g} /$ plate. Inhibition of bacterial growth was observed in $1,000 \mu \mathrm{g} /$ plate in both Salmonella and E. coli. On the other hand, negative results were reported by the National Toxicology Program. ${ }^{15)}$ Carcinogenicity and teratogenicity of MDI have not been reported so far in animals or human subjects. ${ }^{14)}$ The production of this chemical in Japan was 44 million kilograms in 1979 and 70 million kilograms in 1983. ${ }^{12)}$

The carcinogenicity of isobutyl alcohol (IBA) was confirmed in 1975 by Gibel et al., ${ }^{16)}$ but this compound did not demonstrate any mutagenicity. Eight malignant and three benign tumors were produced in 24 Wistar rats by subcutaneous administration of IBA, and three malignant and nine benign tumors were induced in 19 Wistar rats by oral administration. On the other hand, there is a negative report of tumor induction in four rats given IBA orally. ${ }^{17}$

No tumor was produced in mice given 2-propanol (PRO) by subcutaneous injection, inhalation, skin painting ${ }^{18)}$ or oral administration. ${ }^{17)}$ An epidemiological report was published on the incidence of cancer of the paranasal sinuses and the larynx in workers in factories manufacturing PRO by strong acid process. ${ }^{10)}$

Urea is used widely in the chemical industry as a material for nitrogen fertilizers, medicines, celluloid stabilizers, paint, etc., and its output in Japan was 
1.2 million kilograms in $1981 .^{12)}$ Chromosomal aberration of mammalian cells caused by urea was observed in in vitro tests, ${ }^{20)}$ but no DNA damage was noted in alkaline elution/rat hepatocyte assays. ${ }^{21)}$ No mutagenic activity was observed in our mutagenicity test. In spite of the induction of chromosome aberration, no carcinogenicity was observed in rats and mice. ${ }^{22)}$

Methanol did not demonstrate mutagenicity in cell transformation, ${ }^{23)}$ sister chromatid exchange ${ }^{24)}$ or Saccharomyces cerevisiae tests, ${ }^{25)}$ and no carcinogenicity was found in mice, ${ }^{26)}$ rats or dogs. $p$ Xylene is not mutagenic. ${ }^{27-29)}$ 2-Butane, ${ }^{9,30)}$ glycerol, $9,11,28,29) \quad$ 2-ethoxyethanol ${ }^{9,31)}$ and oleic acid $^{9,11,15,17,32,33)}$ were tested for mutagenicity and carcinogenicity with negative results.

Hexanedioic acid showed weak mutagenicity in the host-mediated assay using Saccharomyces $\mathrm{D}_{3}$, but did not present any mutagenicity in in vitro tests using Saccharomyces $\mathrm{D}_{3}$, TA1530 and G46 strains. ${ }^{34)}$ The results of cytogenetic chromosomal aberration test ${ }^{34)}$ and dominant lethal test ${ }^{34)}$ in rats were negative.

2-Methylpropane, a gaseous compound, was not mutagenic to Salmonella, E. coli, mouse lymphoma cells or mouse embryo fibroblasts. ${ }^{35)}$ Carcinogenicity of this compound has not yet been reported.

The carcinogenicity of dodecyl benzene, ${ }^{36)}$ dodecanol, ${ }^{37)}$ iron chloride, ${ }^{9,38)}$ ammonia ${ }^{91}$ and octadecanoic acid ${ }^{9,11,17,38)}$ was tested with negative results.

\section{CONCLUSION}

More than 500 new chemical compounds have been registered at the Ministry of Labor in Japan Since 1979, and obligatory microbial mutation tests have been performed on these chemicals. Testing of the mutagenicity and carcinogenicity of chemicals selected from 45,000 chemicals with consideration given to their structure and annual output have recently been started. The 43 compounds tested by us were registered before 1979 and are manufactured in annual quantities of more than one million kilograms by the chemical industry. Considering the importance of testing the mutagenic activities of these 43 chemicals, using six strains of bacteria according to the Ministry of Labor guidelines because of their high production, our investigation was carried out, and it was found that only two of the 43 compounds were mutagenic.

This work was supported by Japan Chemical Industry Ecology-Toxicology \& Information Center.

\section{REFERENCES}

1) Ames, B.N., McCann, J. and Yamasaki, E: Methods for detecting carcinogens and mutagens with the salmonella/mammalian microsome mutagenicity test. Mutat. Res., 31: 347-364, 1975.

2) Yahagi, T., Nagao, M., Seino, Y., Matsushima, T., Sugimura, T. and Okada, M.: Mutagenicity of N-nitrosoamine of Salmonella, Mutat. Res., 48: 121-130, 1977.

3) McCann, J. and Ames, B.N.: Detection of carcinogens as mutagens in the Salmonella/microsome test: assay of 300 chemicals: discussion, Proc. Natl. Acad. Sci. USA, 73: 950-954, 1976.

4) Sugimura, T., Sato, S., Nagao, M., Yahagi, T. Matsushima, T., Seino, Y., Takeuchi, M. and Kawachi, T.: Overlapping of carcinogens and mutagens. In P.N. Magee (ed.), Fundamentals in Cancer Prevention, 191-215, University of Tokyo Press, Tokyo/Univ. Park Press, Baltimore, 1976.

5) Matsushita, H., Goto, S. and Hamano, Y.: Mutagenicity testing method for volatile compounds, Japan, J. Ind. Health, 23: 761, 1981.

6) U.S. Department of Commerce, National Technical Information Service, PB-274 264: A study of industrial data on candidate chemicals for testing, 3135-138, Environmental Protection Agency, Washington, D.C., 1977.

7) Lijinsky, W. and Taylor, H.W.: Nitrosamines and their precursors in food. In (H.H. Hiat, J.D. Watson and J.A. Winsten, eds.), Origin of Human Cancer; 1579-1590, Cold Spring Harbor Laboratory, University of Tokyo Press, Tokyo, 1977.

8) Watanabe, F. and Sugimoto, S.: Study on the carcinogenicity of aldehyde. 2nd report. Seven cases of transplantable sarcomas of rats appearing in the areas of repeated subcutaneous injection of Urotropin, Gann, 46: 365-367, 1955.

9) Survey of Compounds Which Have Been Tested for Carcinogenic Activity, 2nd edition, Federal Security Agency, Public Health Service, 1951.

10) Della Porta, G., Colnaghi, M.I. and Parmiani, G: Non-carcinogenicity of bexamethylentetramine in mice and rats, Fd. Cosmet. Toxicol., 6: 707-715, 1968.

11) Survey of compounds which have been tested for carcinogenic activity, 1970-1971, U.S. Department of Health, Education and Welfare, Public Health Service, National Cancer Institute of Health. 
Table 5. Mutagenic activities

\begin{tabular}{|c|c|c|c|c|c|c|c|}
\hline \multirow{3}{*}{ No. } & \multirow{3}{*}{ Compound } & \multirow{3}{*}{\multicolumn{2}{|c|}{ Dose }} & \multicolumn{4}{|r|}{ Revertant } \\
\hline & & & & \multicolumn{2}{|c|}{ TA100 } & \multicolumn{2}{|c|}{ TA1535 } \\
\hline & & & & $-\mathbf{S 9}$ & $+\mathbf{S 9}$ & $-\mathbf{S} 9$ & $+\mathrm{S9}$ \\
\hline & Control & & 0 & $191.8 \pm 39.5^{\mathrm{a}}$ & $190.6 \pm 49.3$ & $31.3 \pm 7.0$ & $27.8 \pm 8.4$ \\
\hline & $2 \mathrm{NF}$ & & ug/plate & $700 \pm 52.8$ & $-b$ & - & - \\
\hline & ENNG & 5 & $\prime \prime$ & - & - & $1001.4 \pm 168.5$ & - \\
\hline & $9 \mathrm{AC}$ & 80 & $\prime \prime$ & - & - & - & - \\
\hline \multirow{2}{*}{\multicolumn{2}{|c|}{$\mathbf{B}(\mathrm{a}) \mathbf{P}$}} & & $\prime \prime$ & - & - & - & - \\
\hline & & & $5 \prime \prime$ & - & $956.4 \pm 212.4$ & - & - \\
\hline \multirow{2}{*}{\multicolumn{2}{|c|}{ 2AA }} & 4 & $\prime \prime$ & - & - & - & - \\
\hline & & 2 & $\prime \prime$ & - & - & - & $138.5 \pm 24.8$ \\
\hline \multirow[t]{6}{*}{1.} & \multirow[t]{6}{*}{ Ammonia } & \multicolumn{2}{|c|}{$500 \mathrm{ppm}$} & 243 & 229 & 25 & 31 \\
\hline & & 1,000 & $\prime \prime$ & 251 & 220 & 23 & 40 \\
\hline & & 2,500 & $\prime \prime$ & 198 & 181 & 29 & 35 \\
\hline & & 5,000 & $\prime \prime$ & 207 & 195 & 20 & 21 \\
\hline & & 10,000 & $\prime \prime$ & 197 & 185 & 23 & 34 \\
\hline & & 25,000 & $\prime \prime$ & 227 & 220 & 19 & 31 \\
\hline \multirow[t]{6}{*}{2.} & \multirow[t]{6}{*}{ Butane } & \multicolumn{2}{|c|}{$250 \mathrm{ppm}$} & 216 & 233 & 25 & 28 \\
\hline & & 625 & $\prime \prime$ & 153 & 236 & 24 & 27 \\
\hline & & 1,250 & $\prime \prime$ & 237 & 234 & 25 & 30 \\
\hline & & 2,500 & $\prime \prime$ & 243 & 206 & 20 & 23 \\
\hline & & 5,000 & $" \prime$ & 205 & 233 & 26 & 25 \\
\hline & & 10,000 & $\prime \prime$ & 219 & 254 & 28 & 39 \\
\hline \multirow[t]{6}{*}{3.} & \multirow[t]{6}{*}{ Chlorine } & \multicolumn{2}{|c|}{$0.5 \mathrm{ppm}$} & 93 & 119 & 42 & 43 \\
\hline & & 1.0 & $\prime \prime$ & 92 & 130 & 45 & 43 \\
\hline & & 2.5 & $\prime \prime$ & 92 & 115 & 38 & 37 \\
\hline & & 5.0 & $\prime \prime$ & 111 & 132 & 40 & 35 \\
\hline & & 10.0 & $\prime \prime$ & 120 & 133 & 34 & 41 \\
\hline & & 25.0 & $\prime \prime$ & $82^{*_{\mathfrak{c}}}$ & 133 & $34^{*}$ & 41 \\
\hline \multirow[t]{6}{*}{4.} & \multirow[t]{6}{*}{ 2-Methylpropene } & $250 \mathrm{pl}$ & opm & 180 & 219 & 48 & 27 \\
\hline & & 625 & $\prime \prime$ & 197 & 214 & 53 & 38 \\
\hline & & 1,250 & $\prime \prime$ & 178 & 225 & 52 & 44 \\
\hline & & 2,500 & $\prime \prime$ & 243 & 251 & 43 & 33 \\
\hline & & 5,000 & $\prime \prime$ & 260 & 252 & 37 & 31 \\
\hline & & 10,000 & $\prime \prime$ & 228 & 267 & 28 & 31 \\
\hline
\end{tabular}

ex. $191.8 \pm 39.5^{a}$ : Mean \pm S.D. $-^{\mathrm{b}}$ : not tested. $*_{\mathrm{c}}$ : growth inhibition was observed. 
of 4 volate compounds.

\begin{tabular}{|c|c|c|c|c|c|c|c|}
\hline \multicolumn{2}{|c|}{ WP2 uvrA } & \multicolumn{2}{|c|}{ TA98 } & \multicolumn{2}{|c|}{ TA1537 } & \multicolumn{2}{|c|}{ TA1538 } \\
\hline$-S 9$ & $+\mathbf{S} 9$ & $-\mathbf{S 9}$ & $+\mathbf{S} 9$ & $-\mathbf{S} 9$ & $+\mathrm{S} 9$ & $-\mathrm{S} 9$ & $+\$ 9$ \\
\hline $32 \pm 5.0$ & $28.8 \pm 6.1$ & $48.3 \pm 7.7$ & $60.7 \pm 14.1$ & $11.4 \pm 3.0$ & $10.3 \pm 3.8$ & $31.8 \pm 8.6$ & $58.3 \pm 14.2$ \\
\hline- & 一 & $503.3 \pm 121.9$ & - & - & - & $295.6 \pm 46.5$ & - \\
\hline $136.8 \pm 39.8$ & - & - & 一 & - & - & - & - \\
\hline- & - & - & - & $745 \pm 59.1$ & - & - & - \\
\hline- & - & - & $546 \pm 29.8$ & - & $96.8 \pm 24.6$ & - & $510.5 \pm 183.8$ \\
\hline- & - & - & $389.5 \pm 43.3$ & - & - & - & - \\
\hline- & $132 \pm 50.7$ & - & - & - & - & - & - \\
\hline - & - & - & - & - & - & 一 & - \\
\hline 36 & 31 & 64 & 63 & 11 & 9 & 13 & 37 \\
\hline 32 & 33 & 56 & 76 & 13 & 7 & 27 & 59 \\
\hline 28 & 31 & 43 & 66 & 8 & 9 & 23 & 53 \\
\hline 33 & 28 & 43 & 53 & 10 & 6 & 34 & 55 \\
\hline 33 & 30 & 69 & 60 & 7 & 13 & 32 & 52 \\
\hline 19 & 20 & 57 & 68 & 13 & 10 & 25 & 40 \\
\hline 41 & 42 & 47 & 59 & 10 & 9 & 34 & 52 \\
\hline 41 & 43 & 53 & 65 & 10 & 9 & 30 & 65 \\
\hline 51 & 51 & 64 & 74 & 17 & 29 & 41 & 63 \\
\hline 46 & 61 & 46 & 77 & 22 & 31 & 26 & 58 \\
\hline 33 & 39 & 46 & 56 & 19 & 19 & 34 & 58 \\
\hline 22 & 29 & 52 & 81 & 10 & 14 & 22 & 55 \\
\hline 27 & 26 & 28 & 48 & 12 & 9 & 39 & 65 \\
\hline 29 & 30 & 37 & 55 & 12 & 10 & 33 & 57 \\
\hline 21 & 27 & 38 & 44 & 11 & 10 & 28 & 62 \\
\hline 15 & 27 & 37 & 53 & 11 & 8 & 42 & 67 \\
\hline 11 & 25 & 45 & 52 & 5 & 2 & 32 & 53 \\
\hline 25 & 24 & $13 *$ & $59 *$ & $2^{*}$ & 3 & $27^{*}$ & $52^{*}$ \\
\hline 43 & 40 & 47 & 67 & 12 & 11 & 35 & 55 \\
\hline 44 & 46 & 47 & 77 & 11 & 10 & 34 & 53 \\
\hline 46 & 51 & 67 & 75 & 16 & 21 & 33 & 35 \\
\hline 52 & 62 & 55 & 87 & 18 & 30 & 20 & 52 \\
\hline 32 & 42 & 68 & 84 & 25 & $17^{*}$ & 23 & 56 \\
\hline 28 & 35 & 37 & 92 & $12^{*}$ & $20^{*}$ & 23 & 66 \\
\hline
\end{tabular}


12) Year Book of Chemical Industry's Statistics: 1979-1984. The Ministry of International Trade and Industry, Tokyo.

13) Andersen, M., Binderup, M.L., Kiel, P., Larsen, H. and Maxild, J.: Mutagenic action of isocyanates used in the production of polyurethanes, Scand. J. Work Environ. Health, 6: 221-226, 1980.

14) Woolrich, P.F.: Toxicology, industrial hygiene and medical control of TDI, MDI and PMPPI, Am. Ind. Hyg. Assoc. J., 43: 89-97, 1982.

15) National Toxicology Program of Department of Health and Human Services: NTP Technical Bulletin, No. 6, 1982.

16) Gibel, W., Lohs, Kh. and Wildner, G.P.: Experimentelle Untersuchungen zur kanzerogenen Wirkung von Lösungsmitteln am Beispiel von Propanol-1, 2 methylpropanol-1 und 3-methylbutanol-1, Arch. Geschwulstforsch, 45: 19-24, 1975.

17) Survey of compounds which have been tested for carcinogenic activity. Supplement 2, U.S. Department of Health. Education and Welfare, PHS, NCI, NIH, 1969.

18) Weil, C.S., Smyth, H.F., Jr. and Nale, T.W.: Quest for suspected industrial carcinogen, AMA Arch. Ind. Hyg., 5: 535-547, 1952.

19) IARC monographs on the evaluation of the carcinogenic risk of chemicals to humans, IARC Monogr. Suppl., 4: 151 (IARC, Lyon, France) 1982.

20) Ishidate, M., Sofuni, T. and Yoshikawa, K.: Chromosomal aberration tests in vitro as a primary screening tool for environmental mutagens and/or carcinogens, Gann Monogr., 27: 95-108, 1981.

21) Sina, J.F., Bean, C.L., Dysart, G.R., Taylor, V.I. and Bradley, M.O.: Evaluation of the alkaline elution/rat hepatocytes assay as a prediction of carcinogenic/mutagenic potential, Mutat. Res., 113: 357-391, 1983.

22) Fleischman, R.W., Baker, J.R., Hagopian, M., Wade, G.C., Hayden, D.W., Smith, E.R., Weisburger, J.H. and Weisburger, E.K.: Carcinogenesis bioassay of acetamide, hexanamide, adipamide, urea and $p$ tolylurea in mice and rats, J. Environ. Pathol. Toxicol., 3: 149-170, 1980.

23) Pienta, R.J., Poiley, J.A. and Lebhera, B., III.: Morphological transformation of early passage golden Syrian hamster embryo cells derived from cryopreserved primary cultures as a reliable in vitro bioassay for identifying diverse carcinogens, Int. J. Cancer, 19: 642-655, 1977.

24) Latt, S.A., Allen, J., Bloom, S.E., Carrano, A., Fslke, E., Kram, D., Schneider, E., Schreck, R., Tice, R., Whitfield, B. and Wolff, S.: Sister-chromatid exchange: a report of the Gene Tox Program, Mutat. Res., 87: 17-62, 1981.

25) Abbondandolo, A., Bonatti, S., Corsi, C., Corti, G., Florio, R., Leporini, C., Mazzaccaro, A. and
Nieri, R.: The use of organic solvents in mutagenicity testing, Mutat. Res., 79: 141-150, 1980.

26) Survey of compounds which have been tested for carcinogenic activity, 1974-1975 volume, U.S. Department of Health, and Human Services, PHS, NIH.

27) Bos, R.P., Drouns, R.M.G., von Doorn, R., Theuws, T.L.G. and Henderson, P.TH.: Non mutagenicity of toluene, $o, m$, and $p$-xylene, $o$ methylbenzylalcohol and $o$-methylbenzylsulfate in the Ames assay, Mutat. Res., 88: 273-279, 1981.

28) National Toxicology Program of Department of Health and Human Services: NTP Technical Bulletin, Vol. 1, 1980.

29) Haworth, S., Lawler, T., Mortelmans, K., Speck, W. and Zeiger, E.: Salmonella mutagenicity test results for 250 chemicals, Environ. Mutagen., Supplement 1: 3-142, 1983.

30) Nestmann, E.R., Lee, E.G.H., Matula, T.I., Douglas, G.R. and Mueller, J.C.: Mutagenicity of constituents identified in pulp and paper mill effuents using the Salmonella/mammalian microsome assay, Mutat. Res., 79: 203-212, 1980.

31) National Toxicology Program of Department of Health and Human Services: NTP Technical Bulletin No. 5, 1981.

32) Survey of compounds which have been tested for carcinogenic activity, 1961-1967 volume, U.S. Department of Health, Education and Welfare, PHS, NIH.

33) Survey of compounds which have been tested for carcinogenic activity, 1972-1973 volume, U.S. Department of Health and Human Services, PHS, NIH, Bethesda, Md.

34) Mutagenic evaluation of compound FDA 71-50, Adipic acid, Food and Drug Administration, PB-245 466, 1974, U.S. Department of Commerce, National Technical Information Service.

35) Staab, R.J., and Sarginson, N.J.: Lack of genetic toxicity of isobutylene gas, Mutat. Res., 130: 259260, 1984.

36) Gargus, J.L., Paynter, O.E. and Reese, W.H., Jr.: Utilization of newborn mice in the bioassay of chemical carcinogens, Toxicol. Appl. Pharmacol,, 15: 552-559, 1969.

37) Sice, J.: Tumor-promoting activity of $n$-alkanes and 1-alkanols, Toxicol. Appl. Pharmacol., 9: 70$74,1965$.

38) Nagata, C., Tagashira, Y., Kodama, M., Ioki, Y. and Oboshi, S.: Effect of hydrogen peroxide, Fenton's reagent and iron ions on the carcinogenicity of 3,4 benzopyrene, Gann, 64: 277-285, 1973.

\section{和文要旨}

わが国で生産使用される化学物質のらち，年間 1,000 トン以上の生産量を示す43物質を選び, S. typhimurium 
TA100, TA98, TA1535, TA1537, TA1538 阽よび E. coli WP2 uvrA の6菌株を用いて突然变異原性試験を行っ t.

テストした43物質のらち39物質は，粉末または非揮発 性液体でこのうち2物質のみが陽性を示した，その一 つの hexamethylenetetramine は, TA100および TA 98 に対して S9mix 存在下で, また TA98 では S9mix 非存在下で陽性を示した. つらつつの 4,4'-methylenediphenyldiisocyanate は, TA100 と TA98 の2菌株で S9mix 存在下で陽性を示した.
一方これら43物質の5ち，ガス状または揮発性の高い 4 物質は，ガス暴露法を用いて試験を行ったが，いずれ の結果も䧔性であった．試験をした43物質のうち41物質 が6菌株に対して陰性であったが，文献上なんらかの变 異原性試験結果が報告されているものは11物質 (25.6\%) にすぎない，今回選択した43物質間には化学構造上の類 似性はないが，年間生産量が高く，職業性暴露の可能性 も高いので, 変異原性試験を行い, その結果を報告する ことは有意義であると考える.

Reprint requests to Department of Public Health, The Jikei University School of Medicine, Minato-ku, Tokyo, 105 Japan (H. Shimizu) 著者への通信先 : 清水英做 テ105 東京都港区西新 橋3-25-8 東京慈恵会医科大学公衆葆生学教室 